\title{
Next-generation sequencing of miRNAs and IncRNAs from rat femur and tibia under mechanical stress
}

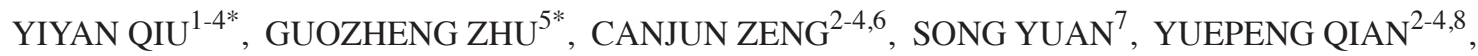 \\ ZELIN YE ${ }^{5}$, SHANWEN ZHAO ${ }^{2-4,6}$ and RUNGUANG LI $^{2-4,6,7}$
}

\author{
${ }^{1}$ Department of Spine Surgery, Section II, Center for Orthopedic Surgery, The Third Affiliated Hospital of \\ Southern Medical University; ${ }^{2}$ Department of Orthopedics, Orthopaedic Hospital of Guangdong Province; \\ ${ }^{3}$ Academy of Orthopedics, Guangdong Province, Guangzhou, Guangdong 510610; ${ }^{4}$ Guangdong Provincial Key Laboratory of \\ Bone and Joint Degenerative Diseases, Guangzhou, Guangdong 510280; ${ }^{5}$ Division of Orthopedics and Traumatology, \\ Department of Orthopedics, Nanfang Hospital, Southern Medical University, Guangzhou, Guangdong 510515; \\ ${ }^{6}$ Department of Foot and Ankle Surgery, Center for Orthopedic Surgery, The Third Affiliated Hospital of \\ Southern Medical University, Guangzhou, Guangdong 510610; ${ }^{7}$ Department of Orthopedics, Linzhi People's Hospital, \\ Linzhi, Tibet 860000; ${ }^{8}$ Department of Pediatric Orthopedics, Center for Orthopedic Surgery, \\ The Third Affiliated Hospital of Southern Medical University, Guangzhou, Guangdong 510610, P.R. China
}

Received November 2, 2020; Accepted May 13, 2021

DOI: $10.3892 / \mathrm{mmr} .2021 .12200$

\begin{abstract}
Exercise intervention has become one of the most effective methods to prevent and treat osteoporosis, which is a common age-related disease and seriously affects the health and quality of life of the elderly. However, the molecular mechanism remains to be elucidated. The present study demonstrated the exercise-induced promotion of osteogenic differentiation and inhibition of adipogenic differentiation in femur and tibia by establishing an animal exercise model using a treadmill exercise system. MicroRNA (miRNA/miR) and long non-coding (lnc)RNA sequencing analyses identified 16 upregulated and two downregulated miRNAs in the exercise group, as well as 44 upregulated lncRNAs and 39 downregulated lncRNAs in the exercise group. There was increased expression of miR-9942 and miR-7704 in both the femur and tibia and an upregulation of miR-30d, miR-5100 and miR-1260 in the femur of animals from the exercise group. In addition, four of the five most downregulated lncRNAs, including lncRNA MSTRG.2625, lncRNA MSTRG.1557, lncRNA MSTRG.691 and IncRNA MSTRG.7497, were demonstrated to be suppressed in both the femur and tibia after treadmill
\end{abstract}

Correspondence to: Dr Runguang Li, Department of Foot and Ankle Surgery, Center for Orthopedic Surgery, The Third Affiliated Hospital of Southern Medical University, 183 Zhongshan Avenue West, Tianhe, Guangzhou, Guangdong 510610, P.R. China

E-mail: lirunguang79@163.com

*Contributed equally

Key words: mechanical stress, treadmill exercise, osteogenic differentiation, microRNA, long non-coding RNA exercise. The results of the present study provided a valuable resource for further exploring the molecular mechanisms underlying the regulation of osteoporosis by exercise.

\section{Introduction}

Exercise intervention, as well as mechanical stress stimulation, has become one of the most effective methods to prevent and treat osteoporosis because exercise has no toxic side effects and possesses osteogenic benefits $(1,2)$. Stress exists in all intracellular environments and can regulate a number of cell and biological functions, such as proliferation and differentiation (3). Mechanical stress stimulation is one of the necessary conditions for maintaining the stability of the skeletal system. A lack of mechanical stimulation will cause bone metabolism disorders, bone microstructure degradation, bone loss and, ultimately, osteoporosis $(4,5)$. Osteoporosis is a common age-related disease, which seriously affects the health and quality of life of the elderly (6). At present, it is considered that possible mechanisms underlying the beneficial effects exercise therapy in prevention and treatment of osteoporosis mainly include the mechanical stimulation effects of exercise, the changes in the levels of hormones and cytokines induced by exercise and the regulation of the signal transduction pathways of bone metabolism (5-7). The exact mechanism of exercise prevention and treatment of osteoporosis, however, has yet to be elucidated. Clarifying the effect of exercise or mechanical stress on bone cells and its mechanism may provide a theoretical basis for further research on the prevention and treatment of osteoporosis by exercise.

MicroRNAs (miRNAs/miRs) are a type of non-coding RNA with a length of 22 nucleotides. Studies have confirmed that miRNAs are widely involved in the regulation of various physiological processes in bone metabolism $(8,9)$. miRNAs can target osteogenic factors, bone resorption factors or other key 
molecules that regulate bone metabolism, and then regulate the proliferation and differentiation of bone marrow mesenchymal stem cells (BMSCs), osteoblasts and osteoclasts (10-12). Previous studies have shown that mechanical stress can also cause the differential expression of miRNAs in BMSCs and osteoblasts, which indicates that miRNAs may be one of the important transducers of exercise or mechanical stress to promote bone formation (13-15). Another type of non-coding RNA with >200 nucleotides, known as long non-coding RNAs (lncRNAs), have also been reported to be able to mediate the regulation of the differentiation of BMSCs (16).

The present study intended to simulate mechanical stress stimulation through treadmill exercise experiments, thus exploring the key miRNAs or lncRNAs mediating osteoblastic differentiation in femur and tibia by using miRNA and lncRNA sequencing and aimed to provide a basis for further research on the molecular mechanisms underlying the regulation of osteoporosis by exercise.

\section{Materials and methods}

Rat exercise model. A total of 12 male 8-week-old Sprague Dawley rats (weight, 280-320 g) provided by Beijing Vital River Laboratory Animal Technology Co., Ltd. were randomly divided into an exercise group and a control group $(\mathrm{n}=6)$. The animals were fed ad libitum with clean food and water under a 12-h light/dark cycle. The constant temperature of the animal room was $20-25^{\circ} \mathrm{C}$ and the relative humidity was $20-25 \%$. The exercise group was treated with 8 weeks of moderate treadmill exercise. In the first 3 days, the initial speed, slope and time were set as $10 \mathrm{~m} / \mathrm{min}$ at a $0^{\circ}$ slope for $20 \mathrm{~min}$, which increased to $20 \mathrm{~m} / \mathrm{min}$ at a $5^{\circ}$ slope for $60 \mathrm{~min}$ after 2 days of rest. The exercise was carried out once a day, 5 days a week for 8 weeks. Apart from treadmill exercise, the rats in the exercise group were free to roam their cages, whereas those in the control group moved freely in their cages all day. In the end of the experiments, rat were euthanized using $100 \% \mathrm{CO}_{2}$ anesthesia using an air displacement rate of $20 \%$ of the chamber volume/min. It was determined that the rats had succumbed when they had stopped breathing, the heart had stopped completely and the pupils were dilated. All rats were successfully sacrificed by $\mathrm{CO}_{2}$. All rat experiments (including euthanasia) were performed between October 2019 and March 2020). Finally, the femur and tibia were collected and isolated for further investigation. All animal experiments were conducted according to relevant national and international guidelines and approved by the Animal Care and Use Committee of Nanfang Hospital, Southern Medical University. The animal protocol no. for these experiments was NFYY-2019-58.

Reverse transcription-quantitative $(R T-q) P C R$. Total RNA was isolated from the femur and tibia using TRIzol ${ }^{\circledR}$ (Thermo Fisher Scientific, Inc.), according to the manufacturer's instructions. Total RNA $(1 \mu \mathrm{g})$ was reverse transcribed using a reverse transcription kit (cat. no. DBI-2220; DBI; http://www. yisonbio.com/yisen001-Products-14675239/), according to the manufacturer's protocol using the following temperature conditions: $42^{\circ} \mathrm{C}$ for $30 \mathrm{~min}$ followed by $80^{\circ} \mathrm{C}$ for $5 \mathrm{~min}$. qPCR was performed using a PCR kit (cat. no. AOPR-1200;
GeneCopoeia, Inc.). The $2^{-\Delta \Delta \mathrm{Cq}}$ method was used for quantification (17). The initial denaturation was at $95^{\circ} \mathrm{C}$ for $10 \mathrm{sec}$, followed by 40 cycles of $55^{\circ} \mathrm{C}$ for $20 \mathrm{sec}$ and $72^{\circ} \mathrm{C}$ for $35 \mathrm{sec}$. $\beta$-actin and $U 6$ were used as internal reference for mRNAs, miRNAs and lncRNAs. The primers (including reference gene) used in RT-qPCR are shown in Tables I and II.

Western blotting. The femur and tibia were lysed using ice-cold RIPA lysis buffer (Fdbio Science) supplemented with protease inhibitor (Fdbio Science) and total proteins were subsequently extracted and quantified using a BCA Protein Assay kit (Fdbio Science). Equal amounts of proteins $(60 \mu \mathrm{g})$ were loaded in each lane, separated via $10 \%$ SDS-PAGE (Biosharp Life Sciences) and then transferred to a PVDF membrane (EMD Millipore). After blocking with 5\% FBS (Gibco; Thermo Fisher Scientific, Inc.) for $2 \mathrm{~h}$ at $25^{\circ} \mathrm{C}$, the membranes was successively incubated with primary antibodies overnight at $4^{\circ} \mathrm{C}$ and secondary antibodies for $2 \mathrm{~h}$ at room temperature. Each membrane was visualized using enhance chemiluminescence reagent (cat. no. 11520709001; Roche Applied Science). Densitometric analysis was performed using ImageJ (version 1.8.0; National Institutes of Health). The antibodies used for western blotting are given in Table III.

Immunofluorescence. Sections of femur and tibia for were fixed for $20 \mathrm{~min}$ with $4 \%$ paraformaldehyde at $25^{\circ} \mathrm{C}$ and the sections permeabilized for another $30 \mathrm{~min}$ at $37^{\circ} \mathrm{C}$ with $0.3 \%$ Triton X-100. After washing with $0.1 \mathrm{M}$ PBS, they were incubated with peroxisome proliferator-activated receptor $\gamma$ (PPAR $\gamma)$ primary antibody (1:400; cat. no. MAB3872; Sigma-Aldrich; Merck KGaA) at $4^{\circ} \mathrm{C}$ overnight. Next, Alexa Fluor 488-conjugated AffiniPure Goat Anti-Rabbit IgG (1:500; cat. no. BF05002, Beijing Biodragon Immunotechnologies Co., Ltd.) was used at room temperature for $90 \mathrm{~min}$ as a secondary antibody. The samples were incubated with DAPI at $25^{\circ} \mathrm{C}$ for $20 \mathrm{sec}$ to stain the nucleus. Fluorescent staining and images were captured in five randomly selected fields using a fluorescence microscope (magnification, x200). The image were processed using NIS-Elements software (V4.2.2; Nikon Corporation) and ImageJ 1.8.0 software (National Institutes of Health; contrast enhancement).

miRNA sequencing. Total RNA was extracted from the femurs and tibias using TRIzol reagent (Thermo Fisher Scientific, Inc.), according to the manufacturer's instructions. The experimental procedure were conducted according to the standard process provided by Illumina, Inc., including the preparation of libraries and sequencing. Small RNA sequencing libraries were prepared using truseq small RNA sample prep kits (Illumina, Inc.). After the preparation of each library, the libraries were sequenced with an Illumina hiseq 2000/2500 (Illumina, Inc.) and the read length was 1x50 bp. Downstream bioinformatics analysis was performed by ACGT101-miR (LC Sciences).

lncRNA sequencing. Total RNA was extracted from the femurs and tibias using TRIzol reagent (Thermo Fisher Scientific, Inc.), according to the manufacturer's instructions. rRNA deletion was used to construct strand-specific 
Table I. Primers (mRNAs) used in reverse transcription-quantitative PCR.

\begin{tabular}{|c|c|c|c|}
\hline Gene & Gene ID & Primer sequence $\left(5^{\prime}-3^{\prime}\right)$ & Length (bp) \\
\hline Runx2 & NM_001145920.2 & $\begin{array}{l}\text { Forward: TGGCTTGGGTTTCAGGTTAG } \\
\text { Reverse: GGTTTCTTAGGGTCTTGGAGTG }\end{array}$ & 104 \\
\hline$P P A R \gamma$ & NM_001127330.2 & $\begin{array}{l}\text { Forward: GAACCTGCATCTCCACCTTATT } \\
\text { Reverse: TGGAAGCCTGATGCTTTATCC }\end{array}$ & 125 \\
\hline$T G F \beta 1$ & NM_011577.2 & $\begin{array}{l}\text { Forward: GGTGGTATACTGAGACACCTTG } \\
\text { Reverse: CCCAAGGAAAGGTAGGTGATAG }\end{array}$ & 103 \\
\hline$T G F \beta R 1$ & NM_009370.3 & $\begin{array}{l}\text { Forward: CCTTGAGTCACTGGGTGTTATG } \\
\text { Reverse: CCACTTAGCTGTCACCCTAATC }\end{array}$ & 117 \\
\hline Smad2 & NM_001252481.1 & $\begin{array}{l}\text { Forward: GCTGAGTGCCTAAGTGATAGTG } \\
\text { Reverse: TACAGCCTGGTGGGATCTTA }\end{array}$ & 103 \\
\hline$\beta$-actin (reference) & NM_007393.5 & $\begin{array}{l}\text { Forward: GAGGTATCCTGACCCTGAAGTA } \\
\text { Reverse: CACACGCAGCTCATTGTAGA }\end{array}$ & 104 \\
\hline
\end{tabular}

Runx2, runt-related transcription factor 2; PPAR $\gamma$, peroxisome proliferator-activated receptor $\gamma ; T G F \beta R 1$, TGF $\beta$ receptor 1 .

libraries for lncRNA sequencing. An Illumina Novaseq ${ }^{\mathrm{TM}}$ 6000 (Illumina, Inc.) was used after each library passed quality inspection. The read length was 2x150 bp (PE150), paired end read length in single channel. After reads were assembled using the transcriptional assembly software stringtie (18), known mRNAs and transcripts $<200$ bp were removed and the remaining transcripts were further subjected to IncRNA prediction. The prediction software used was coding potential calculator (https://github. com/biocoder/cpc) and coding noncoding index (https://github. com/www-bioinfo-org/CNCI\#install-cnci). If remaining transcripts had the potential to encode proteins, they were classified as novel mRNAs and then filtered from the lncRNA dataset. The expression levels of lncRNAs by were measured by fragments per kilobase of exon model per million mapped reads (FPKM) and the expression abundance of known genes in different samples counted by FPKM values.

Analysis of data of miRNA and lncRNA sequencing. Kyoto Encyclopedia of Genes and Genomes (KEGG) was used to analysis the target genes of differentially expressed miRNAs (DEMs), and Gene Ontology (GO) enrichment analysis was carried out for these. The edgerR (Bioconductor software package, https://bioconductor.org/packages/release/ bioc/html/edgeR.html; http://www.R-project.org/) was used for statistical analysis of miRNA sequencing and lncRNA sequencing data: i) Genes with biological duplication and $\mid \log _{2}$ fold changel $\geq 1$ and P-value $\leq 0.05$ were accepted and then counted; and ii) genes without biological duplication and $\log _{2}$ fold changel $\geq 1$ and $\mathrm{P}$-value $<1$ were accepted and then counted.

Statistical analyses. RT-qPCR and western blotting experiments were performed in triplicate and the data were shown as mean \pm SD. Unpaired Student's t-test was used for statistical analysis. Statistical significance (P-value) was calculated by SPSS 17.0 (SPSS, Inc.). $\mathrm{P}<0.05$ was considered to indicate a statistically significant difference.

\section{Results}

Effects of exercise on biomarkers of osteogenesis or adipogenesis and the TGF $\beta 1 /$ Smad2 pathway in femurs and tibias. An exercise rat model was established through treadmill exercise based on previous research $(19,20)$, and the optimization of pre-experiments and corresponding biological indices were measured. The rats were randomly divided into an exercise group and a control group. Rats in the exercise group were treated with 8 weeks of moderate treadmill exercise. Subsequently, the expression levels of runt-related transcription factor 2 (Runx2), a biomarker of osteogenesis (21); PPAR a biomarker of adipogenesis (22); and TGF $\beta 1, T G F \beta R 1$ and Smad2 were detected by qPCR (Fig. 1A-E) and western blotting (Fig. 1F). The results demonstrated that compared with the control group, the femurs and tibias collected from the exercise group demonstrated significantly higher osteogenic activity, represented by an upregulation of Runx2 and lower adipogenic activity, represented by the downregulation of $P P A R \gamma$. In addition, the levels of TGF $\beta 1, T G F \beta R 1$ and Smad 2 were all upregulated in the exercise group, which was consistent with our previous study regarding MSCs loading stress in vitro (23). These results also demonstrated that exercise could promote the osteogenic differentiation while inhibiting their adipogenic differentiation in femur and tibia. In addition, immunofluorescence detection of PPAR $\gamma$ revealed its downregulated expression (Fig. 2). Finally, miRNA and lncRNA sequencing were performed from femurs and tibias.

Exploration of key miRNAs in femur and tibia with mechanical stress by miRNA sequencing. As shown in Fig. 3A, based on the screening criteria of having a $\log _{2}$ fold change $>1$ and $\mathrm{P}<0.05$, 16 miRNAs were upregulated and two miRNAs were downregulated in the exercise group. There were five upregulated miRNAs and one downregulated miRNA identified when $\mathrm{P}<0.01$ (Fig. 3B). Enrichment analysis was performed based on the target genes of the identified DEMs. As shown in Fig. 3C, KEGG analysis demonstrated that target genes of DEMs were 
Table II. Primers (miRNAs and lncRNAs) used in reverse transcription-quantitative PCR.

\begin{tabular}{ll}
\hline Gene (miRNAs) & \\
\hline$m i R-9942-3 p$ & Forward: CGGGCGAGGGCCGGGC \\
& Reverse: CCTGTTGTCTCCAGCCACAAAAGAGCACAATATTTCAGGAGACAACAGGCCCGCCC \\
$m i R-1260$ & Forward: CGCCGATCCCACCGCT \\
& Reverse: CCTGTTGTCTCCAGCCACAAAAGAGCACAATATTTCAGGAGACAACAGGTGGTGGC \\
$m i R-30 d-3 p$ & Forward: CGCCGCTTTCAGTCAGATGT \\
& Reverse: CCTGTTGTCTCCAGCCACAAAAGAGCACAATATTTCAGGAGACAACAGGGCAGCAA \\
$m i R-7704$ & Forward: CGCCGCCGGGGTCGGCG \\
& Reverse: CCTGTTGTCTCCAGCCACAAAAGAGCACAATATTTCAGGAGACAACAGGACATCGC \\
$m i R-5100-p 5$ & Forward: CGCCGATCCCAGCGGT \\
& Reverse: CCTGTTGTCTCCAGCCACAAAAGAGCACAATATTTCAGGAGACAACAGGTGGAGGC \\
F6 & Forward: CTCGCTTCGGCAGCACA \\
& Reverse: AACGCTTCACGAATTTGCGT \\
&
\end{tabular}

Gene (lncRNAs)

Primer sequence $\left(5^{\prime}-3^{\prime}\right)$

$\begin{array}{ll}\text { MSTRG.7497 } & \text { Forward: GTCGTTGGTGGCAGCAG } \\ & \text { Reverse: GGCCGAGCTTAGAACGC } \\ \text { MSTRG.2625 } & \text { Forward: GCCAGAGGTGACCTGTGAAG } \\ & \text { Reverse: TGAACACGAAGGTTTGAGCC } \\ \text { MSTRG.1557 } & \text { Forward: TTTCAGTTCCGCCAATCCAAC } \\ & \text { Reverse: TCTTTCCCATCAGGGTCAGCA } \\ \text { MSTRG.691 } & \text { Forward: CTGCTGCTCCTCTACTGTTCTG } \\ \text { MSTRG.13994 } & \text { Feverse: ACCTTCGTTTGTCTGACTTGC } \\ & \text { Forward: GATTCCCACTGTCCCTACCTA } \\ \beta \text {-actin (reference) } & \text { Feverse: CCTCCCACTTATTCTACACCTC } \\ & \text { Ferward: GCAAGGATACTGAGAGCAAGAG } \\ & \text { Reverse: GGATGGAATTGTGAGGGAGATG }\end{array}$

miRNA, microRNA; lncRNAs, long non-coding RNA.

most significantly enriched in 'pathways in cancer'. GO enrichment analysis, by contrast, demonstrated that the target genes of DEMs were most enriched in the 'positive regulation of transfer' in biological processes, in 'nucleus' and 'integral component of membrane' in the cellular component and in 'protein binding' in terms of molecular functions (Fig. 4A). Finally, five upregulated miRNAs were selected, including miR-9942, miR-30d, miR-7704, miR-5100 and miR-1260, all with $\mathrm{P}<0.01$, for further verification in the femur and tibia by qPCR. The results indicated an increased expression of miR-9942 and miR-7704 in both the femur and tibia, as well as the upregulation of the other three specifically in the femur (Fig. 4B-F).

Exploration of key lncRNAs in femur and tibia with mechanical stress by lncRNA sequencing. As shown in Fig. 5A and B, based on the screening criteria of having a $\log _{2}$ fold change $>1$ and $\mathrm{P}<0.05,44$ lncRNAs were found to be upregulated and 39 IncRNAs to be downregulated in the exercise group. In addition, enrichment analysis was performed based on the identified differentially expressed lncRNAs (DELs). As is shown in Fig. 5C, the most significantly enriched signaling pathways obtained from KEGG analysis was 'systemic lupus erythematosus'. By contrast, the GO enrichment analysis demonstrated that the target genes of DELs were most enriched in 'cell proliferation' in terms of biological processes, in 'extracellular exosome' in terms of cellular component and in 'protein binding' in terms of molecular functions (Fig. 6A). Finally, the five most downregulated lncRNAs, including lncRNA MSTRG.2625, lncRNA MSTRG.1557, lncRNA MSTRG.691, lncRNAMSTRG.7497 and lncRNA MSTRG.13994, were selected for further verification in the femur and tibia by qPCR. The qPCR results were the same as those observed by sequencing, except for IncRNA MSTRG.13994, which further validated the previous enrichment analysis (Fig. 6B-F).

\section{Discussion}

It has been reported that osteoporosis exhibit increased bone marrow fat, while bone mass is reduced (24). To study the mechanism by which exercise improves bone metabolism 
Table III. Antibodies used in western blotting.

\begin{tabular}{|c|c|c|c|c|}
\hline Antibody & Supplier & Cat. no. & Isotype & Dilution \\
\hline Anti-Runx2 & BIOSS & bs-1134R & Rabbit & $1: 500$ \\
\hline Anti-PPAR $\gamma$ & BIOSS & bsm-33436M & Rabbit & $1: 1,000$ \\
\hline Anti-Smad2 & BIOSS & bs-0718R & Mouse & $1: 1,000$ \\
\hline Anti-TGF $\beta 1$ & BIOSS & bs-0103R & Rabbit & $1: 500$ \\
\hline Anti-TGF $\beta$ R 1 & BIOSS & bs-0638R & Rabbit & $1: 1,000$ \\
\hline HRP-Goat Anti-Mouse IgG & Jackson ImmunoResearch Laboratories, Inc. & $115-035-003$ & & $1: 10,000$ \\
\hline HRP-Goat Anti-Rabbit IgG & Jackson ImmunoResearch Laboratories, Inc. & $111-035-003$ & & $1: 10,000$ \\
\hline
\end{tabular}

Runx2, runt-related transcription factor 2; PPAR $\gamma$, peroxisome proliferator-activated receptor $\gamma$; TGF $\beta$ R , TGF $\beta$ receptor 1.
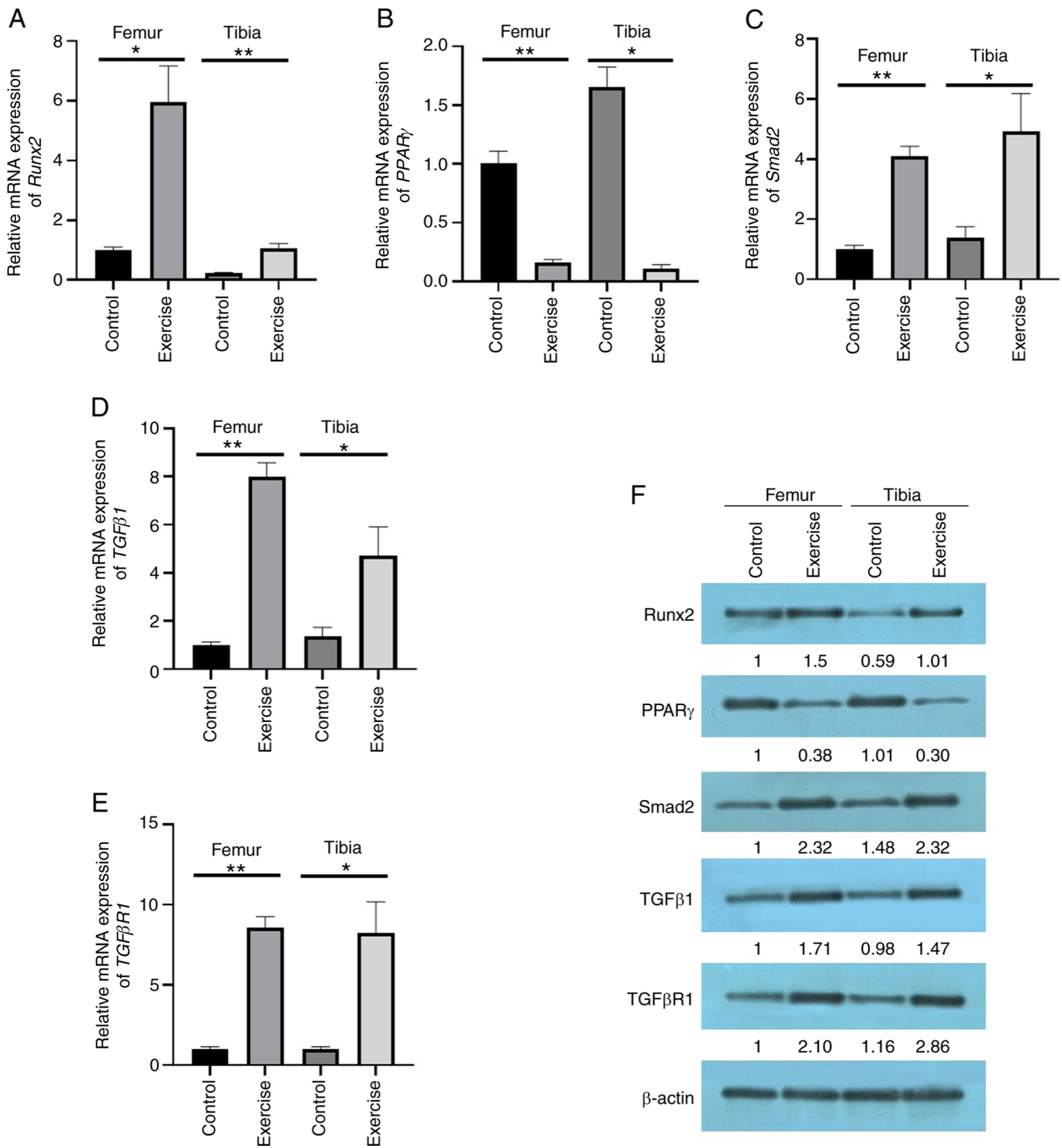

Figure 1. Measurement of biological indices in a rat exercise model. The mRNA expression of (A) Runx2,(B) PPAR $\gamma,(\mathrm{C})$ Smad2, (D) TGF $\beta 1$ and (E) TGF $\beta R 1$ in the femur and tibia of rats in both exercise and control groups was detected by reverse transcription-quantitative PCR. (F) The protein expression of Runx2, PPAR $\gamma$, Smad2, TGF $\beta 1$ and TGF $\beta R 1$ in the femur and tibia of rats in both an exercise and control group was detected by western blotting. Data are presented as the mean $\pm \mathrm{SD}(\mathrm{n} \geq 3),{ }^{*} \mathrm{P}<0.05,{ }^{* *} \mathrm{P}<0.01$ (t-test). Runx2, runt-related transcription factor 2 ; PPAR $\gamma$, peroxisome proliferator-activated receptor $\gamma$; $T G F \beta R 1$, TGF $\beta$ receptor 1 . 


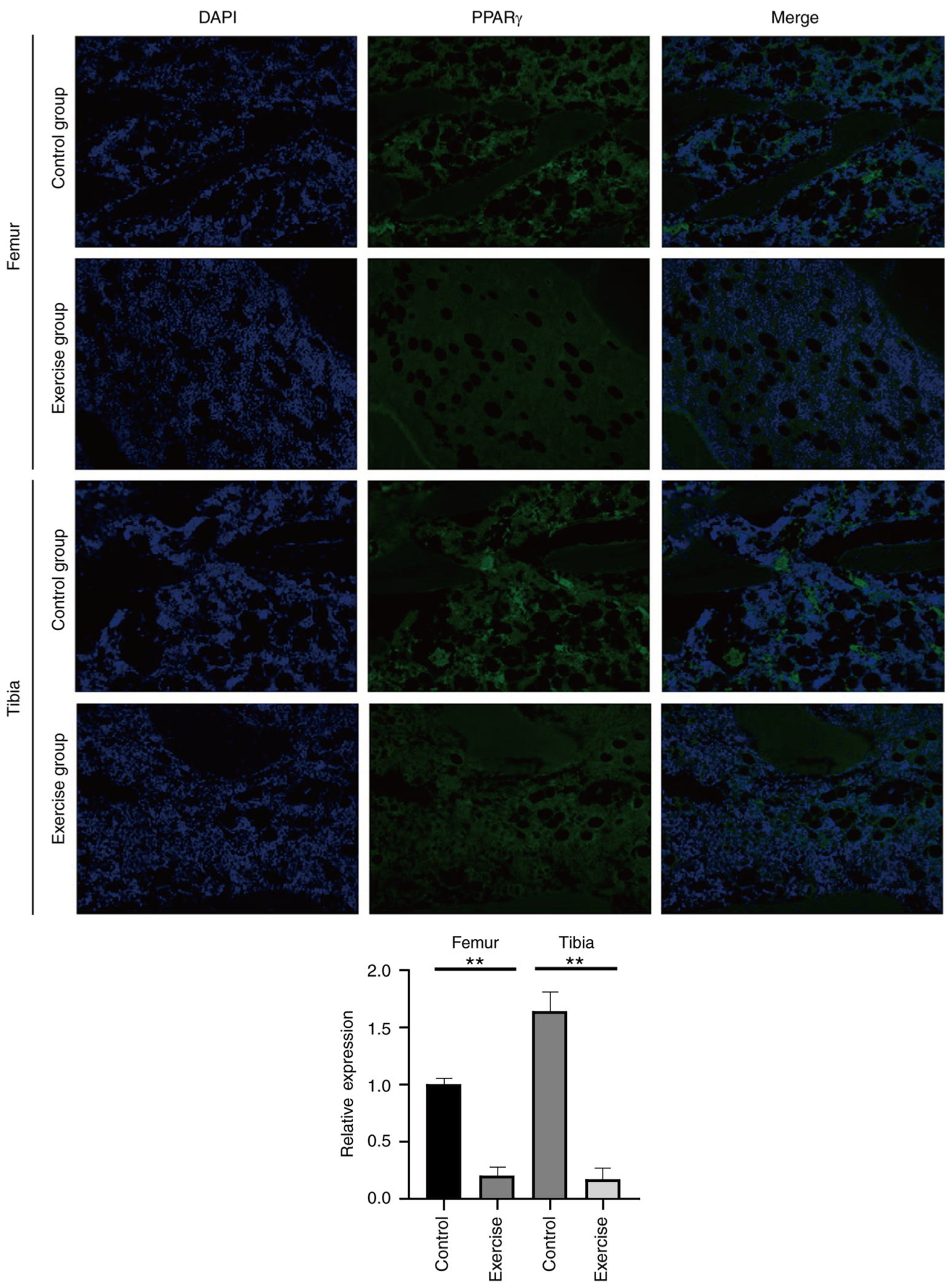

Figure 2. PPAR $\gamma$ in an exercise and control group by immunofluorescence; magnification, $x 200 .{ }^{* *} \mathrm{P}<0.01$. PPAR $\gamma$, peroxisome proliferator-activated receptor $\gamma$.

in more depth, mechanical stress was used to interfere with BMSCs, osteoblasts, osteocytes and osteoclasts, simulating the mechanical stimulation of exercise on the bones. BMSCs are primitive multipotent cells that can differentiate into osteoblasts, adipocytes, chondrocytes and other cells under specific induction conditions (25). BMSCs serve an important role in bone growth and metabolism and age-related osteoporosis and their ability to proliferate and transform into osteoblasts decreases as the body ages, which may be one of the mechanisms driving age-related osteoporosis (26). Some studies have revealed that appropriate mechanical stress stimulation can promote the osteogenic differentiation of BMSCs, inhibit 


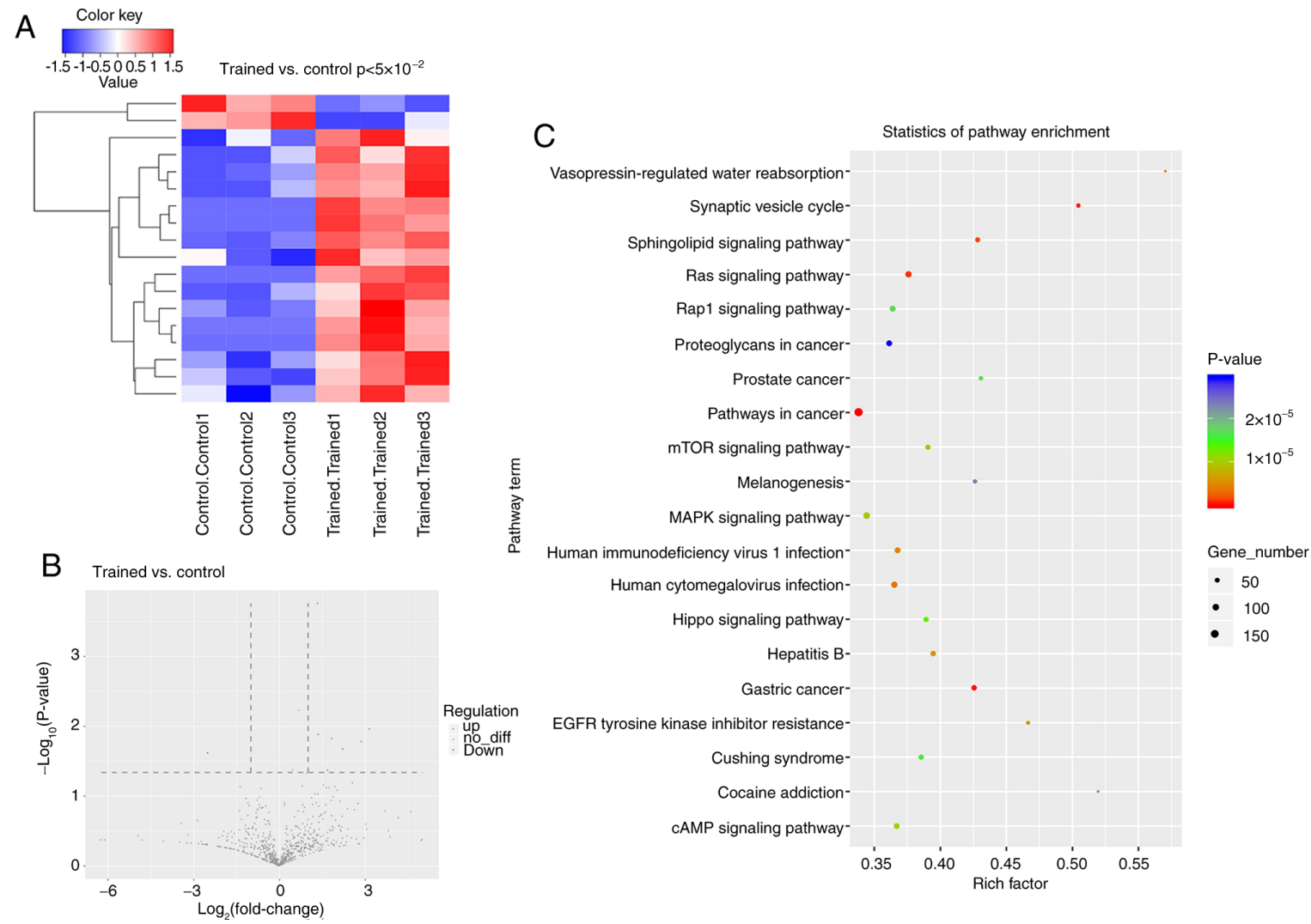

Figure 3. Differentially expressed miRNAs. (A) Heatmap $\left(\mathrm{P}<10^{-2}\right)$ to show differentially expressed miRNAs screened by miRNA sequencing. (B) Volcano plot to show differentially expressed miRNAs $\left(\mathrm{P}<10^{-3}\right)$. (C) Kyoto Encyclopedia of Genes and Genomes signaling pathway enrichment analysis based on differentially expressed miRNAs. (edgerR was used for statistical analysis.). miRNA, microRNA.
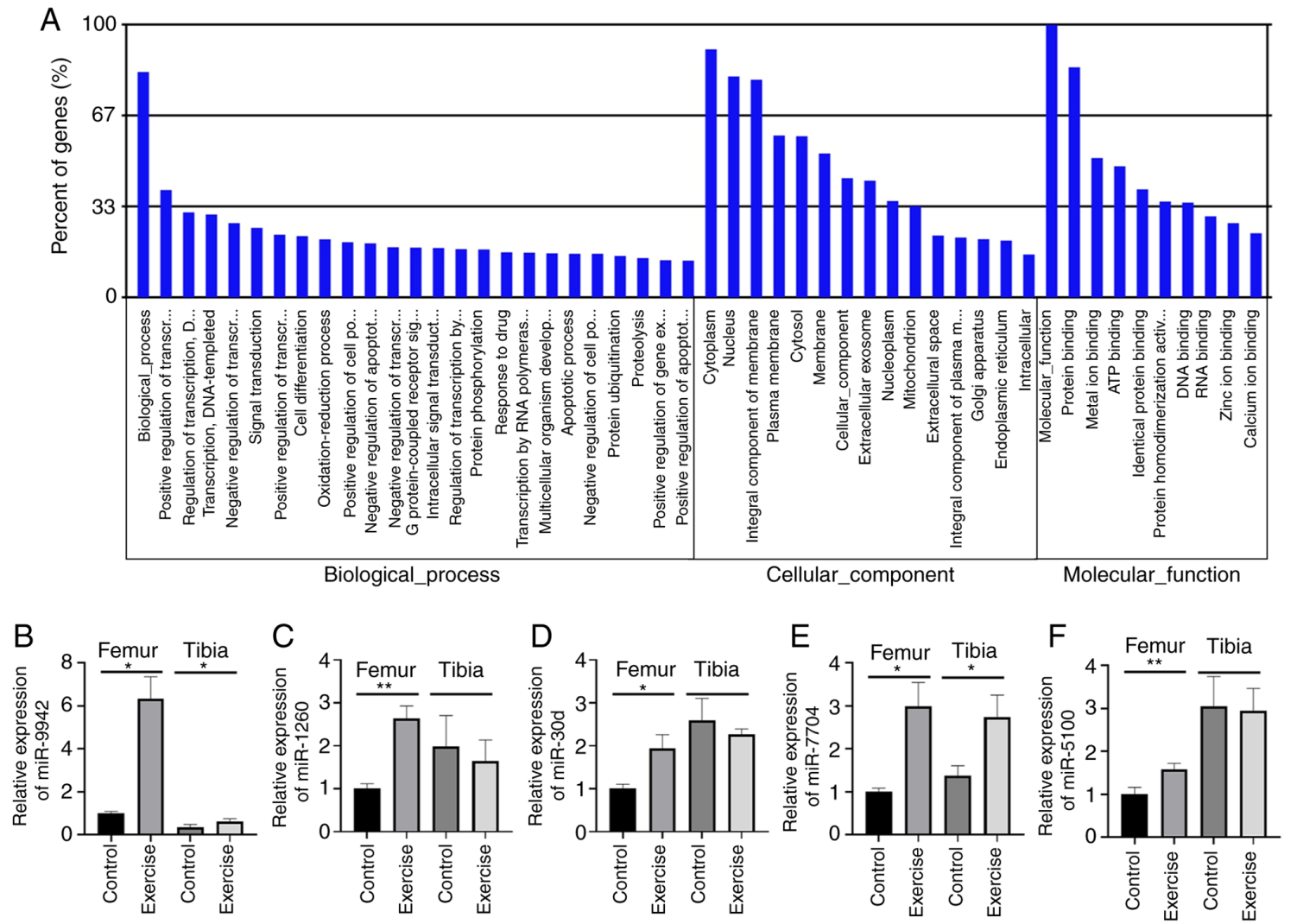

Figure 4. Detection of selected differentially expressed miRNAs. (A) Gene Ontology enrichment analysis was made based on differentially expressed miRNAs. The expression of (B) miR-9942, (C) miR-1260, (D) miR-30d, (E) miR-7704 and (F) miR-5100 in the femur and tibia of rats in both exercise and control groups was detected by reverse transcription-quantitative PCR. Data are presented as the mean $\pm \mathrm{SD}(\mathrm{n} \geq 3),{ }^{*} \mathrm{P}<0.05,{ }^{* *} \mathrm{P}<0.01$ (t-test). miRNA/miR, microRNA. 
A Differentially expressed IncRNAs in different groups

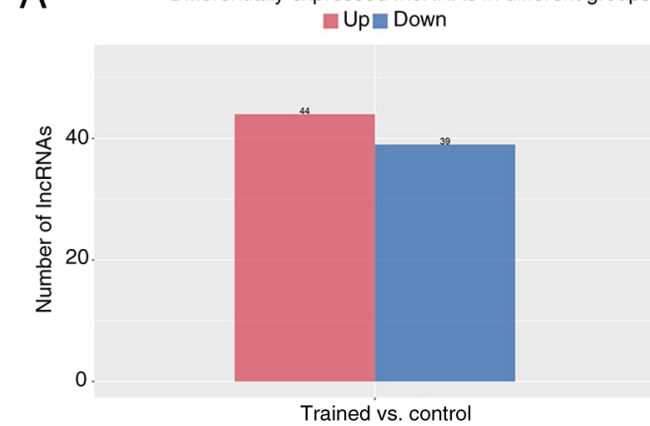

C

B Trained vs. control . Up - No - Down

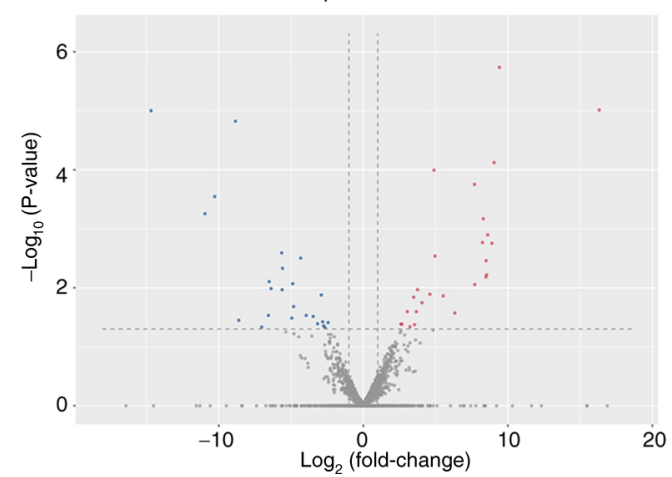
Transcriptional misregulation in cancer Statistic of pathway enrichment Systemic lupus erythematosus Serotonergic synapse Regulation of actin cytoskeleton Ras signaling pathway PPAR signaling pathway One carbon pool by folate Necroptosis Morphine addiction watasa. Hematopoietic cell lineage GABAergic synapse Fc gamma R-mediated phagocytosis ECM-receptor interaction Dopaminergic synapse Circadian entrainment Chemokine signaling pathway Alcoholism African trypanosomiasis Gene_number - 5

- 10

- 15

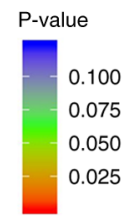

Figure 5. Differentially expressed lncRNAs. (A) Through lncRNA sequencing, 44 upregulated lncRNAs and 39 downregulated lncRNAs were identified. $(\mathrm{P}<0.01)$. (B) Volcano plot to show differentially expressed lncRNAs $(\mathrm{P}<0.01)$. (C) Kyoto Encyclopedia of Genes and Genomes signaling pathway enrichment analysis based on differentially expressed lncRNAs.(edgerR was used for statistical analysis.). lncRNA, long non-coding RNA.

their adipogenic differentiation, promote the proliferation and differentiation of osteoblasts, reduce the activity and number of osteoclasts, promote bone formation and inhibit bone resorption (27-29). In our previous in vitro studies, it has been demonstrated that mechanical stretch could regulate MSCs osteogenic/adipogenic differentiation through the TGF $\beta / \mathrm{Smad}$ signaling pathway and high intensity stress could damage the biological activity of cells due to the imbalance of oxygen free radical system $(23,30-32)$. In order to further explore the molecular mechanisms underlying the regulation of osteoporosis by exercise, animal experiment on mechanical stress are performed in vivo, using a treadmill exercise system. The present study demonstrated the exercise-induced promotion of osteogenic differentiation and inhibition of adipogenic differentiation in femur and tibia and identified 16 upregulated and two downregulated miRNAs in the exercise group, as well as 44 upregulated lncRNAs and 39 downregulated lncRNAs in the exercise group through miRNA and lncRNA sequencing analyses. The results provide a valuable resource for further exploring the molecular mechanisms underlying the regulation of osteoporosis by exercise.

It has been noted that miRNAs mainly target osteogenic factors, bone resorption factors, or other key factors involved in the regulation of bone metabolism to exert their regulatory effects on the proliferation or differentiation of osteoblasts and osteoclasts (14). A type of miRNA can enhance the proliferation and differentiation of osteoblasts and inhibit the proliferation of osteoclasts, thereby promoting bone formation. For example, miR-19a-3p can alleviate the progression of osteoporosis by downregulating the expression of histone deacetylases
(HDACs). HDAC4 can inhibit the function of Runx 2 and miR-19a-3p exerts its osteogenic effects by targeting the expression of HDAC4 (33). Yang et al (34) found that miR-21 could promote the migration and osteogenic differentiation of BMSCs by increasing the activation of the AKT pathway and hypoxia inducible factorl $\alpha$. Huang et al (35) demonstrated that the expression level of miR-488 was significantly lower during the process of psoralen-induced osteogenic differentiation in BMSCs. In addition, experimental verification of bioinformatics analysis and luciferase report analysis identified Runx 2 as a potential target of miR-488, which provides a possible target for the treatment of osteoporosis (36). More specifically, mechanical stress stimulation can upregulate the expression of osteogenic factors, such as ALP, osterix, osteocalcin and Runx2; inhibit the expression of bone resorption markers, such as receptor activator of NF- $\mathrm{NB}$ ligand and tartrate-resistant acid phosphatase; promote the differentiation of mesenchymal stem cells into osteoblasts; and enhance the proliferation and differentiation of osteoblasts via specific miRNA (15). Notably, miRNAs are one of the important molecules for mechanical stress to exert its osteogenesis effects (36). Research by Guo et al (37) demonstrated that mechanical traction can increase ALP activity, upregulate the expression of osteogenic markers and induce the differential expression of miR-218, miR-191, miR-3070a and miR-33. A previous study demonstrated that mechanical stress upregulates the protein expression of Runx2, which promotes the proliferation and differentiation of osteoblasts and also inhibits the expression of miR-103a (38). Additionally, luciferase reporter gene experiments have confirmed that Runx 2 is a target of miR-103a, which indicates that mechanical stress may 
A $\quad$ Biological_process $\square$ Cellular_component $\square$ Molecular_function
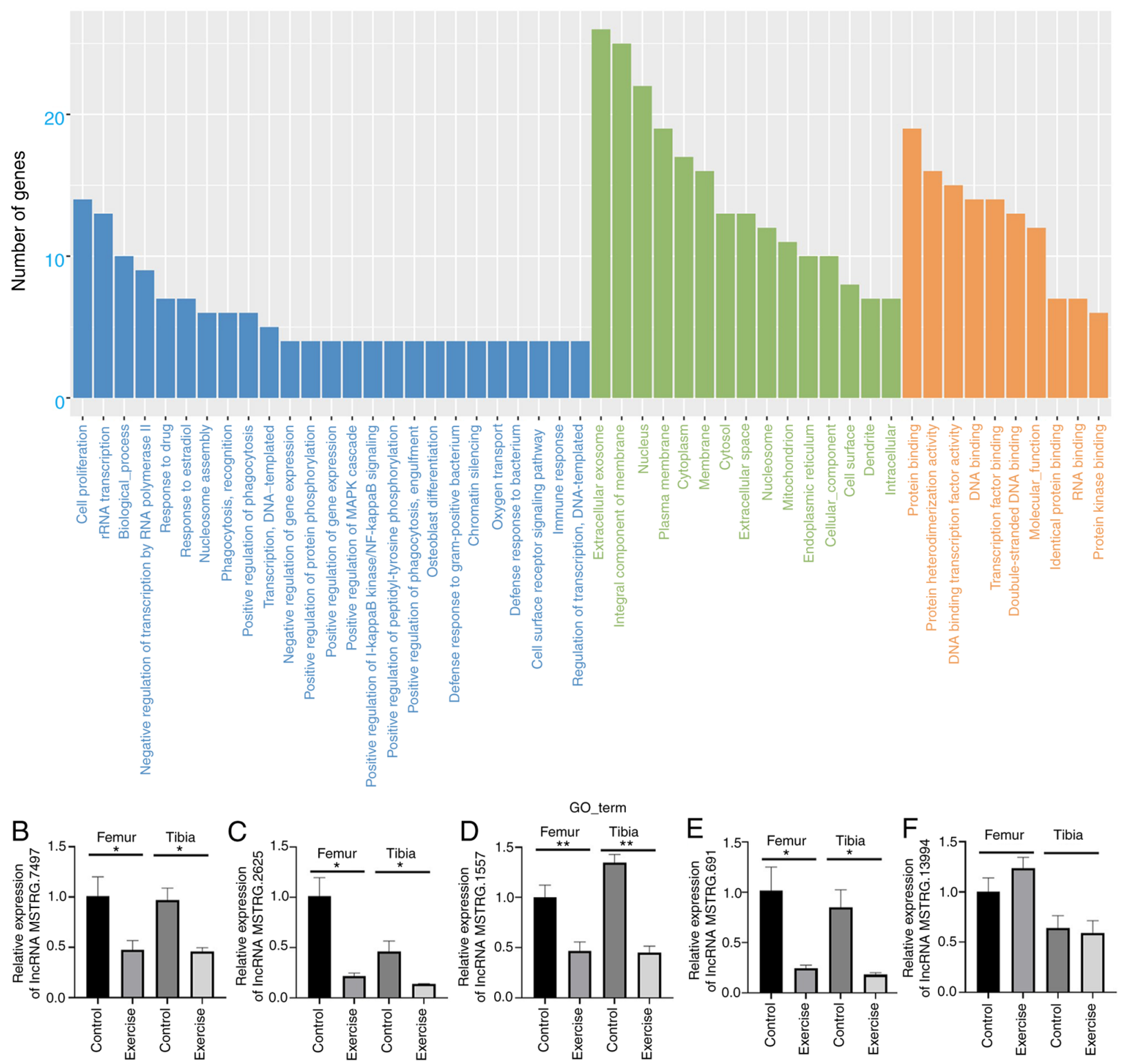

Figure 6. Detection of selected differentially expressed lncRNAs. (A) GO enrichment analysis was made based on differentially expressed lncRNAs. The expression of lncRNA (B) MSTRG.7494, (C) lncRNA MSTRG.2625, (D) lncRNA MSTRG.1557, (E) lncRNA MSTRG.691 and (F) lncRNA MSTRG.13994 in the femur and tibia of rats in both exercise and control groups was detected by reverse transcription-quantitative PCR. Data are presented as the mean \pm SD $(\mathrm{n} \geq 3),{ }^{*} \mathrm{P}<0.05,{ }^{* *} \mathrm{P}<0.01$ (t-test). GO, Gene Ontology. lncRNA, long non-coding RNA.

increase the expression of Runx 2 by inhibiting the expression of miR-103a and thus promote bone formation (38). By contrast, it has been shown that the mechanism of bone metabolism disorder and osteoporosis caused by a long-term lack of mechanical stress stimulation also may be related to miRNA (39). Sun et al (40) studied the proliferation of MC3T3-E1 osteoblasts in a microgravity environment and found that the proliferation of osteoblasts is inhibited when the expression of miR-103 is upregulated, while inhibiting the expression of miR-103 could promote the proliferation of osteoblasts.

Among the five miRNAs identified and verified in the present study as being upregulated in exercise-treated rats, the biological functions of miR-9942 and miR-5100 have not been investigated previously to the best of the authors' knowledge. It has been noted that miR-30d-3p is significantly upregulated during the differentiation of pancreatic stem cells into $\beta$ cells, may serve an important role in the epigenetic mechanism of $\beta$ cell differentiation and could be useful in the diagnosis and treatment of diabetes (41). It has been reported that the expression of miR-7704 increases in hepatocellular carcinoma and liver cirrhosis and exerts a negative regulatory effect on lncRNA HAGLR (42). In addition, Siglecl overexpression increases the expression of miR-1260, thus degrading $\mathrm{I} \kappa \mathrm{B} \alpha$ through its untranslated region to serve a pro-inflammatory role and promote the progression of chronic obstructive pulmonary disease (43).

Accumulating evidence has shown that lncRNAs also serve an important role in the regulation of osteogenic differentiation and adipogenic differentiation of BMSCs, occasionally acting as competing endogenous RNAs to regulate miRNAs. For 
example, lncRNA KCNQ1OT1 and lncRNA MSC-AS1 were found to be able to upregulate BMP2 thus promoting the osteogenic differentiation of BMSCs by sequestering miR-214 and miR-140-5p, respectively $(44,45)$. Previous studies reveal that IncRNAMEG3 is involved in the regulation of differentiation and metabolism of mesenchymal stem cell by interacting and regulating miRNAs. For example, IncRNA MEG3 can regulate the adipogenic and osteogenic differentiation of human adipose stem cells through miR-140-5p, while the osteogenic differentiation of human periodontal ligament stem cells in periodontitis was affected through the miR-27a-3p/IGF1 axis $(46,47)$. Notably, IncRNAs also can act as a mediator in the regulation of BMSC differentiation. For example, lncRNA H19 acts as a competing endogenous RNA for miR-138 to upregulate the expression level of downstream focal adhesion kinase, thereby acting as a positive regulator of the BMSC osteogenic differentiation induced by mechanical stress stimulation (48).

The lack of BMD MRI measurements of bone content and strength are the limitations of the present study.

In summary, considering the key roles served by miRNAs and IncRNAs in the differentiation, the present study established an animal model through treadmill exercise experiments and performed miRNA and lncRNA sequencing to identify differentially expressed miRNAs and lncRNAs in femur and tibia that may be key mediators of differentiation. The results of the present study are a valuable resource for further exploring the molecular mechanisms driving the development of osteoporosis and allow the search for therapeutic targets.

\section{Acknowledgements}

Not applicable.

\section{Funding}

This work was supported by the National Natural Sciences Foundation of China (grant no. 81101366) and the Natural Science Foundation of Guangdong Province (grant nos. 2018A030313640 and 2019A1515012176).

\section{Availability of data and materials}

The datasets generated and/or analyzed during the current study are available in the NCBI's Gene Expression Omnibus repository (https://www.ncbi.nlm.nih.gov/geo/query/acc. cgi?acc $=$ GSE168713).

\section{Authors' contributions}

RL designed this program. YQ, GZ, SY and YQ operated the cell and animal experiments. YQ, GZ, CZ, ZY and SZ conducted the data acquisition and analysis. GZ and $\mathrm{CZ}$ uploaded all the data onto an online repository. RL, YQ, GZ and $\mathrm{CZ}$ examined the revised the paper. YQ, GZ, CZ and RL produced the manuscript, which was checked by RL and $\mathrm{YQ}$. $\mathrm{RL}$ and $\mathrm{YQ}$ are responsible for confirming the authenticity of the raw data. All authors read and approved the final manuscript.

\section{Ethics approval and consent to participate}

All animal experiments were conducted according to relevant national and international guidelines and approved by the Animal Care and Use Committee of Nanfang Hospital, Southern Medical University. The animal protocol no. for these experiments was NFYY-2019-58.

\section{Patient consent for publication}

Not applicable.

\section{Competing interests}

The authors declare that they have no competing interests.

\section{References}

1. Christianson MS and Shen W: Osteoporosis prevention and management: Nonpharmacologic and lifestyle options. Clin Obstet Gynecol 56: 703-710, 2013.

2. Todd JA and Robinson RJ: Osteoporosis and exercise. Postgrad Med J 79: 320, 2003.

3. Matamoro-Vidal A and Levayer R: Multiple influences of mechanical forces on cell competition. Curr Biol 29: R762-R774, 2019.

4. Hemmatian H, Bakker AD, Klein-Nulend J and van Lenthe GH: Aging, osteocytes, and mechanotransduction. Curr Osteoporos Rep 15: 401-411, 2017.

5. Sandino C, McErlain DD, Schipilow J and Boyd SK: Mechanical stimuli of trabecular bone in osteoporosis: A numerical simulation by finite element analysis of microarchitecture. J Mech Behav Biomed 66: 19-27, 2017.

6. Yuan Y, Chen X, Zhang L, Wu J, Guo J, Zou D, Chen B, Sun Z Shen $\mathrm{C}$ and Zou J: The roles of exercise in bone remodeling and in prevention and treatment of osteoporosis. Prog Biophys Mol Biol 122: 122-130, 2016.

7. Tong X, Chen X, Zhang S, Huang M, Shen X, Xu J and Zou J: The effect of exercise on the prevention of osteoporosis and bone angiogenesis. Biomed Res Int 2019: 8171897, 2019.

8. Mohr AM and Mott JL: Overview of microRNA biology. Semin Liver Dis 35: 3-11, 2015.

9. Rupaimoole R and Slack FJ: MicroRNA therapeutics: Towards a new era for the management of cancer and other diseases. Nat Rev Drug Discov 16: 203-222, 2017.

10. Chapurlat RD and Confavreux CB: Novel biological markers of bone: From bone metabolism to bone physiology. Rheumatology 55: 1714-1725, 2016.

11. Taipaleenmäki H: Regulation of bone metabolism by microRNAs. Curr Osteoporos Rep 16: 1-12, 2018.

12. Tang P, Xiong Q, Ge W and Zhang L: The role of microRNAs in osteoclasts and osteoporosis. Rna Biol 11: 1355-1363, 2014.

13. Yuan Y, Guo J, Zhang L, Tong X, Zhang S, Zhou X, Zhang M, Chen X, Lei L, Li H, et al: MiR-214 attenuates the osteogenic effects of mechanical loading on osteoblasts. Int J Sports Med 40: 931-940, 2019.

14. Wang J, Liu S, Li J, Zhao S and Yi Z: Roles for miRNAs in osteogenic differentiation of bone marrow mesenchymal stem cells. Stem Cell Res Ther 10: 197, 2019.

15. Yuan Y,Zhang L, Tong X, Zhang M, Zhao Y, Guo J, Lei L, Chen X, Tickner J, Xu J and Zou J: Mechanical stress regulates bone metabolism through MicroRNAs. J Cell Physiol 232: 1239-1245, 2017.

16. Wang X, Zhao D, Zhu Y, Dong Y and Liu Y: Long non-coding RNA GAS5 promotes osteogenic differentiation of bone marrow mesenchymal stem cells by regulating the miR-135a-5p/FOXO1 pathway. Mol Cell Endocrinol 496: 110534, 2019.

17. Barra GB, Santa Rita TH, Almeida ALSC, Jácomo RH and Nery LFA: Serum has higher proportion of janus kinase 2 V617F mutation compared to paired EDTA-whole blood sample: A model for somatic mutation quantification using qPCR and the $2^{-\Delta \Delta C q}$ method. Diagnostics (Basel) 10: 153, 2020.

18. Pertea M, Kim D, Pertea GM, Leek JT and Salzberg SL: Transcript-level expression analysis of RNA-seq experiments with HISAT, StringTie and Ballgown. Nat Protoc 11: 1650-1667, 2016. 
19. Tamaki H, Akamine T, Goshi N, Kurata H and Sakou T: Effects of exercise training and etidronate treatment on bone mineral density and trabecular bone in ovariectomized rats. Bone 23 : 147-153, 1998

20. Kannus P, Sievänen H, Järvinen TL, Järvinen M, Kvist M, Oja P, Vuori I and Jozsa L: Effects of free mobilization and low- to high-intensity treadmill running on the immobilization-induced bone loss in rats. J Bone Miner Res 9: 1613-1619, 1994.

21. Bruderer M, Richards R, Alini M and Stoddart M: Role and regulation of RUNX2 in osteogenesis. Eur Cells Mater 28: 269-286, 2014

22. Ali AT, Hochfeld WE, Myburgh R and Pepper MS: Adipocyte and adipogenesis. Eur J Cell Biol 92: 229-236, 2013.

23. Li R, Liang L, Dou Y, Huang Z, Mo H, Wang Y and Yu B: Mechanical stretch inhibits mesenchymal stem cell adipogenic differentiation through TGF $\beta 1 / \mathrm{Smad} 2$ signaling. J Biomech 48 : 3665-3671, 2015

24. Wang C, Meng H, Wang X, Zhao C, Peng J and Wang Y: Differentiation of bone marrow mesenchymal stem cells in osteoblasts and adipocytes and its role in treatment of osteoporosis. Med Sci Monit 22: 226-233, 2016.

25. Naji A, Eitoku M, Favier B, Deschaseaux F, Rouas-Freiss N and Suganuma N: Biological functions of mesenchymal stem cells and clinical implications. Cell Mol Life Sci 76: 3323-3348, 2019.

26. Infante A and Rodríguez CI: Osteogenesis and aging: Lessons from mesenchymal stem cells. Stem Cell Res Ther 9: 244, 2018.

27. Menuki K, Mori T, Sakai A, Sakuma M, Okimoto N, Shimizu Y, Kunugita $\mathrm{N}$ and Nakamura T: Climbing exercise enhances osteoblast differentiation and inhibits adipogenic differentiation with high expression of $\mathrm{PTH} / \mathrm{PTHrP}$ receptor in bone marrow cells. Bone 43: 613-620, 2008.

28. Lee J, Park H and Kim KS: Intrinsic and extrinsic mechanical properties related to the differentiation of mesenchymal stem cells. Biochem Biophys Res Commun 473: 752-757, 2016.

29. Fu X, Halim A, Tian B, Luo Q and Song G: MT1-MMP downregulation via the PI3K/Akt signaling pathway is required for the mechanical stretching-inhibited invasion of bone-marrow-derived mesenchymal stem cells. J Cell Physiol 234: 14133-14144, 2019.

30. Li R, Liang L, Dou Y, Huang Z, Mo H, Wang Y and Yu B Mechanical strain regulates osteogenic and adipogenic differentiation of bone marrow mesenchymal stem cells. Biomed Res Int 2015: 873251, 2015.

31. Li R, Chen B, Wang G, Yu B, Ren G and Ni G: Effects of mechanical strain on oxygen free radical system in bone marrow mesenchymal stem cells from children. Injury 42: 753-757, 2011

32. Zhu G, Qian Y, Wu W and Li R: Negative effects of high mechanical tensile strain stimulation on chondrocyte injury in vitro. Biochem Biophys Res Commun 510: 48-52, 2019.

33. Chen R, Qiu H, Tong Y, Liao F, Hu X, Qiu Y and Liao Y: MiRNA-19a-3p alleviates the progression of osteoporosis by targeting HDAC4 to promote the osteogenic differentiation of hMSCs. Biochem Biophys Res Commun 516: 666-672, 2019.

34. Yang C, Liu X, Zhao K, Zhu Y, Hu B, Zhou Y, Wang M, Wu Y, Zhang $\mathrm{C}, \mathrm{Xu}$ J, et al: miRNA-21 promotes osteogenesis via the PTEN/PI3K/Akt/HIF-1 $\alpha$ pathway and enhances bone regeneration in critical size defects. Stem Cell Res Ther 10: 65, 2019.

35. Huang Y, Hou Q, Su H, Chen D, Luo Y and Jiang T: miR-488 negatively regulates osteogenic differentiation of bone marrow mesenchymal stem cells induced by psoralen by targeting Runx 2 . Mol Med Rep 20: 3746-3754, 2019.
36. Wei F, Yang S and Wang S: MicroRNAs: A critical regulator under mechanical force. Histol Histopathol 33: 335-342, 2018.

37. Guo Y, Wang Y, Liu Y, Liu Y, Zeng Q, Zhao Y, Zhang X and Zhang X: MicroRNA-218, microRNA-191*, microRNA-3070a and microRNA-33 are responsive to mechanical strain exerted on osteoblastic cells. Mol Med Rep 12: 3033-3038, 2015.

38. Zuo B, Zhu J, Li J, Wang C, Zhao X, Cai G, Li Z, Peng J, Wang P, Shen C, et al: microRNA-103a functions as a mechanosensitive microRNA to inhibit bone formation through targeting Runx2. J Bone Miner Res 30: 330-345, 2015.

39. Blaber EA, Dvorochkin N, Torres ML, Yousuf R, Burns BP, Globus RK and Almeida EA: Mechanical unloading of bone in microgravity reduces mesenchymal and hematopoietic stem cell-mediated tissue regeneration. Stem Cell Res 13: 181-201, 2014.

40. Sun Z, Cao X, Hu Z, Zhang L, Wang H, Zhou H, Li D, Zhang S and Xie M: MiR-103 inhibits osteoblast proliferation mainly through suppressing Cav1.2 expression in simulated microgravity. Bone 76: 121-128, 2015.

41. Coskun E, Ercin M and Gezginci-Oktayoglu S: The role of epigenetic regulation and pluripotency-related MicroRNAs in differentiation of pancreatic stem cells to beta cells. J Cell Biochem 119: 455-467, 2018

42. Mahlab-Aviv S, Boulos A, Peretz AR, Eliyahu T, Carmel L, Sperling R and Linial M: Small RNA sequences derived from pre-microRNAs in the supraspliceosome. Nucleic Acids Res 46: 11014-11029, 2018.

43. Li S, Jiang L, Yang Y, Cao J, Zhang Q, Zhang J, Wang R, Deng $X$ and Li Y: Siglec1 enhances inflammation through miR-1260-dependent degradation of I $\mathrm{B} \alpha$ in COPD. Exp Mol Pathol 113: 104398, 2020.

44. Wang CG, Liao Z, Xiao H, Liu H, Hu YH, Liao QD and Zhong D: LncRNA KCNQ1OT1 promoted BMP2 expression to regulate osteogenic differentiation by sponging miRNA-214. Exp Mol Pathol 107: 77-84, 2019.

45. Zhang N, Hu X, He S, Ding W, Wang F, Zhao Y and Huang Z: LncRNA MSC-AS1 promotes osteogenic differentiation and alleviates osteoporosis through sponging microRNA-140-5p to upregulate BMP2. Biochem Biophys Res Commun 519: 790-796, 2019.

46. Li Z, Jin C, Chen S, Zheng Y, Huang Y, Jia L, Ge W and Zhou Y: Long non-coding RNA MEG3 inhibits adipogenesis and promotes osteogenesis of human adipose-derived mesenchymal stem cells via miR-140-5p. Mol Cell Biochem 433: 51-60, 2017.

47. Liu Y, Liu C, Zhang A, Yin S, Wang T, Wang Y, Wang M, Liu Y, Ying Q, Sun J, et al: Down-regulation of long non-coding RNA MEG3 suppresses osteogenic differentiation of periodontal ligament stem cells (PDLSCs) through miR-27a-3p/IGF1 axis in periodontitis. Aging (Albany NY) 11: 5334-5350, 2019.

48. Wu J, Zhao J, Sun L, Pan Y, Wang H and Zhang W: Long non-coding RNA H19 mediates mechanical tension-induced osteogenesis of bone marrow mesenchymal stem cells via FAK by sponging miR-138. Bone 108: 62-70, 2018.

This work is licensed under a Creative Commons Attribution-NonCommercial-NoDerivatives 4.0 International (CC BY-NC-ND 4.0) License. 